\title{
The Australasian Radiopharmaceutical Trials Network: Clinical Trials, Evidence, and Opportunity
}

\author{
Roslyn J. Francis ${ }^{1}$, Dale L. Bailey ${ }^{2}$, Michael S. Hofman ${ }^{3}$, and Andrew M. Scott ${ }^{4}$ \\ ${ }^{1}$ Sir Charles Gardiner Hospital and University of Western Australia, Perth, Australia; ${ }^{2}$ Department of Nuclear Medicine at Royal North \\ Shore Hospital and Faculty of Medicine and Health at University of Sydney, Sydney, Australia; ${ }^{3}$ Molecular Imaging and Therapeutic \\ Nuclear Medicine at Peter MacCallum Cancer Centre and Sir Peter MacCallum Department of Oncology at University of Melbourne, \\ Melbourne, Australia; and ${ }^{4}$ Department of Molecular Imaging and Therapy at Austin Health, Olivia Newton-John Cancer Research \\ Institute and School of Cancer Medicine at La Trobe University, and Faculty of Medicine at University of Melbourne, Melbourne, \\ Australia
}

$\mathbf{R}$ patient care. As we enter this ero to ensure that our specialty is well equipped to assess, evaluate, and provide evidence for the clinical application of new imaging and therapeutic agents to translate from research or experimental applications to clinical practice. The challenge for nuclear medicine is how to obtain evidence of efficacy, cost effectiveness, and clinical indications, in order to progress from initial studies to an approved, government- or insurance-funded imaging or therapeutic agent. This is particularly relevant for imaging studies and therapies that are not developed by companies and when academic sites participate in investigator-initiated multicenter trials aiming to develop evidence for regulatory approval.

The initial development of evidence for ${ }^{18} \mathrm{~F}$-FDG PET was one of the first examples of multicenter prospective academic trials aimed at generating data for regulatory approval. In 2001, the Australian PET Data Collection project was initiated to generate evidence of the clinical impact of PET and the changes in management introduced by PET findings. This multicenter prospective trial program evaluated over 30,000 consecutive patients undergoing ${ }^{18}$ F-FDG PET across the country over a 2 -y period, and trials evaluating the management impact on oncology patients produced compelling data leading to Medicare approval of many clinical indications (1). In the United States, the National Oncologic PET Registry was established in 2006 to evaluate data on the clinical utility of PET through a prospective data registry and not only enabled the Centers for Medicare and Medicaid Services to support broad funding for PET in oncology but also provided compelling data on clinical utility (2). In addition, the Society of Nuclear Medicine and Molecular Imaging established its Clinical Trials Network in 2008 to assist with developing new molecular imaging radiopharmaceuticals through scanner validation, clinical protocols, and training programs (3). These programs are examples of how clinical evidence

Received Nov. 26, 2020; revision accepted Dec. 14, 2020.

For correspondence or reprints, contact Andrew M. Scott (andrew.scott@ austin.org.au).

Published online Dec. 31, 2020.

COPYRIGHT (C) 2021 by the Society of Nuclear Medicine and Molecular Imaging. DOI: 10.2967/jnumed.120.258152 can be developed for nuclear medicine studies by academic groups. The challenge that has more recently emerged is that regulatory bodies require that health technology assessments contain more detailed clinical and economic outcomes before new imaging and therapeutic radiopharmaceuticals can be approved; hence, new approaches to generating these levels of evidence are required.

To address the need for a more comprehensive clinical trials program in nuclear medicine in Australia, the Australasian Radiopharmaceutical Trials Network (ARTnet) was formed in 2014. This was a joint initiative between the 2 professional nuclear medicine organizations, the Australian and New Zealand Society of Nuclear Medicine and the Australasian Association of Nuclear Medicine Specialists. ARTnet was launched with the following mission statement: "To promote and facilitate innovative collaborative clinical research utilising radiopharmaceuticals for imaging or therapy." ARTnet's scientific committee incorporates nuclear medicine specialists, technologists, radiopharmaceutical scientists, and physicists. ARTnet has implemented a broad-reaching program of credentialing scanners, validating sites, defining radiopharmaceutical standard operating procedures, interpreting images centrally, and reviewing clinical trial protocols.

PET and SPECT camera accreditation is an essential element of multicenter trials in which imaging datasets will be analyzed. The importance of this element was highlighted with the PET camera accreditation using ${ }^{68} \mathrm{Ga}$ in preparation for the proPSMA clinical trial (4). The accreditation process used an IEC/NEMA-NU2 body phantom and standardized imaging instructions and uncovered an unanticipated issue with a large underestimate in ${ }^{68} \mathrm{Ga}$ SUV parameters that was eventually found to be related to a factory-shipped incorrect dose calibrator setting (5). Had this accreditation not been undertaken, there would have been an average of $15 \%$ variation in SUV parameters across sites in the trial, which would not be acceptable. ARTnet has also developed radiopharmaceutical production and quality control manuals for clinical trials and has an accreditation process for sites that produce radiopharmaceuticals in-house.

ARTnet provides quality control support and enabling procedures for clinical trials; however, the clinical trials are run as investigator-initiated studies, are led by principal investigators, and are funded by the government, charitable organizations, or sponsored research grants. Trials either are nuclear medicine-led or are 
performed collaboratively in close cooperation with other medical specialty and clinical trials groups.

The formation of ARTnet corresponded to the emergence of PSMA (prostate-specific membrane antigen) PET imaging, and this has been the focus of the initial successful ARTnet-supported studies and publications. The first collaborative ARTnet-supported study was on the management impact of PSMA PET imaging. This questionnaire-based study assessed management intent before and after PSMA PET imaging and demonstrated a 51\% management change in the overall cohort and $62 \%$ in those patients who underwent PSMA PET for biochemical relapse (6). The clinical impact and influence on subsequent treatment decisions are important considerations in the funding of a new imaging test and provide input into cost analysis considerations.

The proPSMA study was the first large imaging trial in which ARTnet was involved in site credentialing and study conduct. ProPSMA was a multicenter prospective randomized clinical trial of ${ }^{68}$ Ga-PSMA PET imaging in 302 men who had newly diagnosed prostate cancer with high-risk features and were being considered for definitive treatment. This study was conducted at 10 clinical trial sites across Australia and completed recruitment ahead of schedule. The results showed that ${ }^{68}$ Ga-PSMA PET brought about a $27 \%$ improvement in accuracy, compared with staging with bone scans and contrast CT, and that PSMA PET was associated with higher reporter agreement and a lower radiation dose than the current standard of care (4). This landmark study provided comprehensive clinical evidence of the superiority of ${ }^{68}$ Ga-PSMA PET over conventional imaging in primary staging of prostate cancer.

ARTnet has provided site accreditation for radiopharmaceutical synthesis and PET camera performance in a series of academic, investigator-initiated multicenter prospective trials of ${ }^{177} \mathrm{Lu}$-PSMA in prostate cancer patients. The TheraP trial (7) was a multicenter prospective randomized phase II clinical trial comparing ${ }^{177} \mathrm{Lu}-$ PSMA versus cabazitaxel in men with metastatic castrate-resistant prostate cancer progressing after docetaxel. There were 291 patients screened, with 200 eligible patients randomized to either ${ }^{177}$ Lu-PSMA or cabazitaxel, from 11 sites around Australia. The study demonstrated a significantly higher PSA-50 (PSA reduction of greater than $50 \%$ from baseline) response rate in those assigned ${ }^{177} \mathrm{Lu}-\mathrm{PSMA}$ than in those assigned cabazitaxel (65/99 [66\%; 95\% CI, 56-75] vs. 37/101 [37\%; 95\% CI, 27-46]; $P<0.001)$, with less grade III/IV toxicity in the ${ }^{177}$ Lu-PSMA group than in the group receiving conventional chemotherapy (8). ARTnet is providing similar support for 2 further randomized phase II clinical trials with ${ }^{177}$ Lu-PSMA, the UpFront PSMA (NCT04343885) and ENZAp (NCT04419402) clinical trials.

Multicenter imaging clinical trials just commencing in which ARTnet is involved include the FIG study $\left({ }^{18} \mathrm{~F}\right.$-FET $\left[O-\left(2-{ }^{18} \mathrm{~F}-\mathrm{flu}-\right.\right.$ oroethyl)-L-tyrosine] in glioblastoma; ACTRN12619001735145), which is a multicenter prospective clinical trial of 210 patients at 10 sites designed to establish the role of ${ }^{18}$ F-FET PET in distinguishing pseudoprogression from true tumor progression or tumor recurrence and the impact on radiotherapy planning. This study will be supported by ARTnet for PET camera accreditation, radiopharmaceutical accreditation for sites undertaking in-house production, and assistance in centralized ${ }^{18} \mathrm{~F}$-FET imaging review and analysis. Another study that is being coordinated by ARTnet is a prospective evaluation of a new quantitative SPECT bone reconstruction algorithm based on incorporating the CT scan into the reconstruction process compared with current state-of-the-art ordered-subsets expectation-maximization reconstruction with resolution recovery.

Collaboration has been pivotal in the success of the current multicenter trials in Australia. There is the opportunity for nuclear medicine sites to participate in multicenter trials, thereby gaining experience with new imaging or therapeutic agents, and resulting in patients gaining access to these studies. ARTnet also provides a mechanism for young and less experienced researchers to become involved in clinical trials. We feel that this joint venture has been the success that it has to date because it was initiated and remains managed by the nuclear medicine clinician researchers who have created a multidisciplinary approach to research design and have been extremely willing to engage with other clinical trial groups. ARTnet has also been approached by other medical specialty clinical trial groups to give input into and participate in diagnostic and therapy-focused multicenter trials. Finally, there is also the opportunity for the ARTnet model to be extended to other countries, which could provide opportunities for broader collaborative networks in nuclear medicine clinical trials.

\section{DISCLOSURE}

Andrew Scott is supported by NHMRC investigator fellowship 1177837. The ProPSMA study was sponsored by the Peter MacCallum Cancer Centre with funding from Movember and the Prostate Cancer Foundation (PCF). The TheraP study was sponsored by the ANZUP Cancer Trials Group with funding from Endocyte (now a Novartis company), Australian Nuclear Science and Technology Organisation, Prostate Cancer Foundation of Australia, Movember, Can4Cancer and It's a Bloke Thing Foundation. The FIG study is supported by the Medical Research Future Fund, Cure Brain Cancer Foundation, and the Australian Brain Cancer Mission. No other potential conflict of interest relevant to this article was reported.

\section{REFERENCES}

1. Scott AM, Gunawardana DH, Kelley B, et al. PET changes management and improves prognostic stratification in patients with recurrent colorectal cancer: results of a multicenter prospective study. J Nucl Med. 2008;49:1451-1457.

2. Hillner BE, Liu D, Coleman RE, et al. The National Oncologic PET Registry (NOPR): design and analysis plan. J Nucl Med. 2007;48:1901-1908.

3. Sunderland JJ, Christian PE. Quantitative PET/CT scanner performance characterization based upon the Society of Nuclear Medicine and Molecular Imaging Clinical Trials Network oncology clinical simulator phantom. J Nucl Med. 2015;56:145-152.

4. Hofman MS, Lawrentschuk N, Francis RJ, et al. Prostate-specific membrane antigen PET-CT in patients with high-risk prostate cancer before curative-intent surgery or radiotherapy (proPSMA): a prospective, randomised, multicentre study. Lancet. 2020;395:1208-1216.

5. Bailey DL, Hofman MS, Forwood NJ, et al. Accuracy of dose calibrators for ${ }^{68} \mathrm{Ga}$ PET imaging: unexpected findings in a multicenter clinical pretrial assessment. J Nucl Med. 2018;59:636-638.

6. Roach PJ, Francis R, Emmett L, et al. The impact of ${ }^{68}$ Ga-PSMA PET/CT on management intent in prostate cancer: results of an Australian prospective multicenter study. J Nucl Med. 2018;59:82-88.

7. Hofman MS, Emmett L, Violet J, et al. TheraP: a randomised phase 2 trial of ${ }^{177} \mathrm{Lu}-$ PSMA-617 theranostic treatment vs carbazitaxel in progressive castration-resistant prostate cancer (clinical trial protocol ANZUP 1603). BJU Int. 2019;124:5-13.

8. Hofman MS, Emmett L, Sandhu SK, et al. TheraP: a randomised phase II trial of ${ }^{177}$ Lu-PSMA-617 (LuPSMA) theranostic versus cabazitaxel in metastatic castration resistant prostate cancer (mCRPC) progressing after docetaxal-initial results (ANZUP protocol 1603) [abstract]. J Clin Oncol. 2020;38(suppl):5500. 